Voix et Images

\title{
Gabrielle Roy : la somme de l'oeuvre
}

\section{Réjean Robidoux}

Volume 14, numéro 3 (42), printemps 1989

Gabrielle Roy

URI : https://id.erudit.org/iderudit/200790ar

DOI : https://doi.org/10.7202/200790ar

Aller au sommaire du numéro

Éditeur(s)

Université du Québec à Montréal

ISSN

0318-9201 (imprimé)

1705-933X (numérique)

Découvrir la revue

Citer cet article

Robidoux, R. (1989). Gabrielle Roy : la somme de l'oeuvre. Voix et Images, 14(3),

376-379. https://doi.org/10.7202/200790ar d'utilisation que vous pouvez consulter en ligne.

https://apropos.erudit.org/fr/usagers/politique-dutilisation/ 


\section{Gabrielle Roy: la somme de l'œuvre}

\section{par Réjean Robidoux, Université d'Ottawa}

Devant le phénomène Gabrielle Roy cinq ans après la mort de l'écrivain, c'est un sentiment de totalité que j'ai personnellement le besoin d'éprouver. Parce que le devenir est définitivement achevé et que, sur le plan du corpus, un grand fonds déjà exploitable se trouve constitué, l'auteur s'offre à nous comme un monument à trois dimensions qu'on doit explorer au dedans comme au dehors et dont il importe de détailler le centre et le pourtour, dans la perspective nouvelle d'une connaissance intégrale, acquise non plus à la pièce, dans le hasard et l'imprévu d'une production en cours, mais vue désormais sous l'angle de l'irrévocable et du déterminé.

Cela implique et signifie que voici le moment d'entreprendre la publication d'une édition novissima, peut-être même double et géminée. Je connais en effet vaguement le projet des administrateurs du Fonds Gabrielle-Roy' ${ }^{1}$ de mettre sur pied une édition «définitive» de l'œuvre, destinée au grand public. Mais je veux aussi me faire le truchement d'un autre projet au long cours, assurément complémentaire, qui tente pour l'instant de prendre forme et qui viserait à produire, d'ici la fin du siècle, une édition à la fois scientifique et complète de Gabrielle Roy. Il s'agira, entre autres, de décider au départ de ce qui devra entrer dans la dénomination globale de tout l'œuvre: les ouvrages que l'auteur a elle-même publiés, certes, et des ébauches importantes qui devront être soigneusement reconstituées (je pense en particulier à la fameuse saga esquissée, façonnée, abandonnée et plusieurs fois reprise, que je me représente fort bien préparée à la façon que l'a été pour la Pléiade le Lieutenant-colonel de Maumort de Roger Martin du Gard2). Mais les écrits divers des premières années de la carrière, les reportages au-delà de ceux qu'on a repris dans Fragiles Lumières de la terre, ou bien les billets et nouvelles des débuts, qui peuvent être classés parmi des juvenilia et qui néanmoins, sous un aspect de genèse, présentent au moins autant d'intérêt que les prolégomènes de tout grand auteur. Et la corrrespondance?... Tout est à entreprendre, tout est à faire, et le comité de direction du programme Corpus d'éditions critiques qui s'apprête à présenter au Conseil canadien de recherches en sciences humaines une deuxième phase quinquennale (et qui en planifie une troisième en prospective) entend consacrer une part importante de ses ressources à la promotion d'un tel projet d'édition critique des œuvres complètes de Gabrielle

1 Corporation privée sans but lucratif administrant la succession littéraire de Gabrielle Roy.

2 Roger Martin du Gard, le Lleutenant-colonel de Maumort, édition établie par André Daspre, Paris, Gallimard, 1983, lv-1316 p. (Bibliothèque de la Pléiade). 
Roy, et j'imagine que plusieurs, voulant profiter des avantages et de l'efficacité assurés dans ce programme, pourront être intéressés à y participer.

Je crois qu'il est opportun et essentiel à ce stade de tenir que l'œuvre capitale de Gabrielle Roy, ce n'est pas Bonheur d'occasion, non plus qu'Alexandre Chenevert, ni la Montagne secrète, ni le cycle fictivomémorialiste, ni l'autobiographie, mais toute l'œuvre, tout l'œuvre, encore en partie inédit et génétiquement toujours mal connu.

Quand on veut dégager de cet ensemble imposant ce que Charles Mauron appelle une structure de grands fonds, on se heurte tout de suite à l'ostensible dichotomie d'un double corpus marqué, d'une part, par le réalisme social qui culmine dans Bonheur d'occasion, constituant un massif de litterature que je qualifierais sommairement de positive, avant tout engagée dans le combat du vivre et bien ancree dans le concret individuel ou collectif. Ce point de force qui, d'entrée de carrière, a littéralement valu à Gabrielle Roy la notoriété et la gloire a peut-être êté aussi à plus ou moins long terme, jusqu'à nos jours, dans un certain contexte, sinon une véritable pierre d'achoppement, du moins une sorte de prétexte et de faux-fuyant pour mésinterpréter le reste plus subtil et, à mon sens, supérieur, plus original et plus universel de l'œuvre. J'y reviendrai.

Ainsi se dessine donc, d'autre part, une masse considérable d'écrits d'un esprit bien différent, plus difficile à étiqueter selon les catégories reçues, où il faut franchement reconnaître ce qui s'appelle du lyrisme idyllique et de l'utopie (expressions de François Ricard), où se déploie en tout cas à loisir et à dessein, consciemment et consciencieusement, la subjectivité de l'imagination autobiographique, dans un apparent repli sur soi-même et l'abstraction esthétique.

Cette partie de l'œuvre, qui a vite fait d'être matériellement et temporellement prépondérante, est justement aussi le lieu d'une interrogation sur la démarche artistique parachevant dans la création de beauté une réflexion symbolique sur la littérature comme justification ou rachat de la vie. Depuis l'étape de la Petite Poule d'eau et d'Alexandre Chenevert, dans la lancée du grand cycle autoreprésentatif et personnel: Rue Deschambault, la Route d'Altamont, Cet été qui chantait, Ces enfants de ma vie, De quoi t'ennuiestu, Éveline?, intégrant encore emblématiquement la Rivière sans repos et Un jardin au bout du monde, avec au centre mathématique de la carrière la Montagne secrète et au milieu de ce récit le propos clef: to tell my story, jusqu'à l'accomplissement à découvert de l'autobiographie dans la Détresse et l'enchantement, Gabrielle Roy pose de façon expresse et constante le choix du raconter contre le vivre, et cela me semble être l'une des leçons fondamentales de l'œuvre. C'est là le ressort primordial de la création régienne.

Or, voir cela en opposition irréconciliable, voire en contradiction, avec l'autre mouvement qui produit Bonheur d'occasion, en y cherchant surtout une cassure et un amoindrissement, ne rend pas du tout justice à la valeur distinctive et organique de l'œuvre intégrale. Et ce n'est d'ailleurs pas ainsi qu'on a voulu en disposer. Je n'invente rien ici: François Ricard, en écrivant son dense petit 
livre $^{3}$ au milieu des années 1970 , connaissait à l'évidence toute l'œuvre jusquelà faite, même le non publié ou ce qui se trouvait dispersé dans des périodiques, et il voyait en outre assez bien venir la suite pour que son étude, qui doit évidemment, après quinze ans, être complétée, ne paraisse d'aucune façon périmée ni dépassée.

Je dirai à présent que les articles subséquents de François Ricard ${ }^{4}$, en 1984, à coups d'arguments historiques - son essai se dit expressément «biographique» - confirment cette profonde vérité régienne, en faisant en l'occurrence toute la lumière sur la supposée cassure, sur quoi précisément il ne faut pas se méprendre, entre Bonheur d'occasion et la Petite Poule d'eau. Il y a eu effectivement vers 1948 une crise d'identité qu'on doit aussi bien reconnaître comme une crise de vocation. L'illumination que François Ricard avait presque suggérée au début de son livre de 1975, en référence à rien moins que Pascal, Descartes ou Valéry, on la trouve donc dûment attestée à la date de ce jour d'été 1949, dans l'environnement inspiré de la cathédrale de Chartres': l'appel est impérieux et détermine un choix radical. On a l'impression, quasiment, écrit François Ricard, que la vie cesse, qu'il ne se passe plus rien, que le mouvement s'arrête, et que seule compte désormais l'écriture, où va se résumer dès lors toute l'activité de Gabrielle Roy. ${ }^{6} \mathrm{Et}$ pareille pulsion, accordée à une foncière fascination du sujet pour sa propre aventure - autobiographique donc constituera l' inspiration dominante, quasi exclusive, de l'écrivain?.

J'ouvre ici à ce propos une parenthèse peut-être tout à fait farfelue. J'ai cru trouver chez Gabrielle Roy un gage symbolique, inconscient mais concret, d'une telle compulsion, lorsqu'elle raconte avoir fixé en elle-même à jamais, au moment de sa visite, seule, à la National Gallery de Londres, au même temps, somme toute, que l'illumination de Chartres, le portrait d'Arnolfini et sa femme que je ne cesse, dit-elle, de revoir d chaque jour de ma vie (DE, p. 347). Au fait, je m'imagine qu'elle devait conserver parmi ses pretiosa une reproduction du célèbre Van Eyck. On sait que ce tableau, avec son petit miroir convexe au centre, qui rend présent en abyme le peintre en personne, est le modèle par excellence, en tout cas, très révélateur de l'œuvre spéculaire.

Quoi qu'il en soit d'une telle hypothèse, ce choix d'avant 1950 est d'une importance déterminante. J'y reconnais un acte véritablement fondateur de destin. Mais j'y vois sous un autre rapport la simple officialisation, désormais sûre, d'une décision prise dix ans plus tôt et qui comportait concomitamment la

3 Gabrielle Roy, Montréal, Fides, 1975, 192 p. (Ecrivains canadiens d'aujourd'hui).

4 «La métamorphose d'un écrivain: essai biographique», Études littéraires, vol. XVII, no 3, hiver 1984, p. 441-455; «Les mémoires secrets d'une jeune fille pas très rangée», l'Actualité, Montréal, octobre 1984, p. 15-18.

5 La lettre du 13 juin 1949 de Gabrielle Roy à sa soeur Bernadette permet de préciser le moment (juin 1949) de cette illumination. Voir Gabrielle Roy, Ma chère petite sour, lettres à Bernadette 1943-1970, édition préparée par François Ricard, Montréal, Boréal, 1988, p. 33.

6 François Ricard, «Les mémoires secrets...», p. 16.

7 François Ricard, "La métamorphose...», p. 453. 
rupture avec le vivre: famille, amitiés, patrie, métier $^{8}$, et, dans le ráconter, le retour salutaire à l'origine, aux commencements, aux débuts $d u$ monde 9 . C'est pourquoi par exemple Mélina Roy, la mère quittée, perdue et retrouvée, n'est pas présente seulement par une dédicace dans la texture de Bonheur d'occasion. La conversion à la réalité ${ }^{10}$, conséquente au pari (dans le sens pascalien du mot) de 1937-1939, et qui aboutit, après d'infinies recherches, errances, ouvertures et culs-de-sac de toutes sortes, au premier sommet romanesque est, envers et contre tout, l'entrée dans le raconter essentiel, obscurément cohérent.

En veine, décidément, d'analogies prestigieuses, je hasarde sans vergogne un rapprochement avec le cas de Proust dont je dirai que l'intentionnalité du Temps retrouvé inspire et informe, par une sorte d'effet rétroactif, l'œuvre entière, non seulement depuis Du côté de chez Swann, mais bien en-deçà des Contre Sainte-Beuve et autres Jean Santeuil, jusqu'aux premiers Désirs et les jours au moins...

Mais je reviens sur terre. Au stade présent de la connaissance de Gabrielle Roy, il ne faudrait pas ignorer ni non plus minimiser l'émergence récente d'une opposition, que je qualifierai de viscérale, à l'œuvre dans son ensemble et au message régiens. Cela a éclaté au grand jour, autant que je puisse savoir, au moment d'un colloque dans l'Ouest, à Saint-Boniface, en novembre 198411. Il s'agit d'une vue sociologisante d'obédience goldmannienne qui réduit l'œuvre et l'entreprise de Gabrielle Roy à ce que l'on nomme une romance canadiennefrançaise. À partir, me semble-t-il, d'un grief plus ou moins scandalisé contre le non-engagement (ou contre le dégagement) politique, contre l'égocentrisme et contre la prétention à l'humanisme pur de l'écrivain, on met en question l'originalité formelle de l'œuvre et l'on déprécie même ce qui paraît le plus ouvert sur le vivre social: Bonheur d'occasion.

Ce n'est pas ici le lieu pour moi d'essayer de rectifier une telle perspective. Mais cela devra quelque jour être fait, et bien à propos. Je m'en tiens pour l'instant à proclamer l'importance de Gabrielle Roy et à plaider, si nécessaire, en faveur de la réalisation du plan d'œuvres vraiment complètes du «Corpus d'éditions critiques».

8 François Ricard, «Les mémoires secrets...», p. 16.

9 François Ricard, «La métamorphose...», p. 450.

10 Ibid., p. 443.

11 Jean Morisset, «Entre la détresse et le déchirement; nature et signification de l'œuvre de Gabrielle Roy: entrevue réalisée avec Miodrag Kapetanovich et Paul Dubé à Saint-Boniface (Manitoba), le 24 novembre 1984», dans la Langue, la Culture et la Société des francophones de l'Ouest, Centre d'études francocanadiennes de l'Ouest, 1985, p. 235-251. Cette publication des actes d'un colloque fait aussi mention (p. 254) d'un texte intitulé Une romance canadienne-française par Myo Kapetanovich, non inclus dans le présent recueil, mais dont on dit qu'il doit être publié par les éditions Nouvelle Optique. 